\title{
Predicting Anisotropic Thermal Displacements from Solid-State NMR
}

Giovanna Pope giovanna.P@knights.ucf.edu

James Harper james.harper@ucf.edu

NMR crystallography methods can now provide crystal structures for certain molecules that cannot be solved by conventional methods. One deficiency, however, in nearly all NMRderived crystal structures is the inability to estimate errors in the atomic positions (i.e. provide the "thermal ellipsoids"). Here an approach is described for estimating errors in $\mathrm{COOH}$ hydrogens in four phases of palmitic acid. Individual hydrogen variations are predicted based on agreement between experimental and DFT computed ${ }^{13} \mathrm{COOH}$ chemical shift tensors. Ultimately, two types of structures are found - those with localized $\mathrm{COOH}$ hydrogens and those having dynamically disordered protons. This work provides the first crystal structure for three of the phases studied. The structure of the fourth phase was previously reported and provides an invaluable comparison of our methodology. For each structural type, uncertainties are determined and compared to benchmark data from single crystal $x$-ray and single crystal neutron diffraction data. 